\title{
NONCONVEX VARIATIONAL PROBLEMS WITH GENERAL SINGULAR PERTURBATIONS
}

\author{
NICHOLAS C. OWEN
}

\begin{abstract}
We study the effect of a general singular perturbation on a nonconvex variational problem with infinitely many solutions. Using a scaling argument and the theory of $\Gamma$-convergence of nonlinear functionals, we show that if the solutions of the perturbed problem converge in $L^{1}$ as the perturbation parameter goes to zero, then the limit function satisfies a classical minimal surface problem.
\end{abstract}

Introduction. Let $f:[0, \infty) \times \mathbb{R} \rightarrow \mathbb{R}$ satisfy

$$
\begin{gathered}
f(p, u) \in C^{2}, \\
f_{p}(0, u)=0 \quad \forall u \in \mathbb{R},
\end{gathered}
$$

there exists $k_{1}>0$ such that $f(p, u) \geq k_{1} p^{2} \quad \forall p \in[0, \infty), \forall u \in \mathbf{R}$,

$$
f_{p p}(p, u)>0 \quad \forall p \in[0, \infty), \forall u \in \mathbf{R},
$$

$f(0, u) \geq 0 \forall u \in \mathbb{R}$, with equality if and only if $u=a$ or $b(-\infty<$ $a<b<\infty)$, and $f_{u u}(0, a) \neq 0, f_{u u}(0, b) \neq 0$,

Let $\Omega$ be a bounded, open subset of $\mathbf{R}^{2}$ with Lipschitz continuous boundary. Define a sequence of functionals, $I_{\varepsilon}$, by

$$
I_{\varepsilon}(u)=\int_{\Omega} f(\varepsilon|\nabla u|, u) d x,
$$

where $\nabla u=\left(\partial u\left(x_{1}, x_{2}\right) / \partial x_{1}, \partial u\left(x_{1}, x_{2}\right) / \partial x_{2}\right)$ and $\varepsilon>0$.

For a fixed $c \in(a, b)$, consider the regular calculus of variations problem:

$\left(P_{\varepsilon}\right)$ Minimize $I_{\varepsilon}$ on $A=\left\{u \in W^{1,1}(\Omega): \int_{\Omega} u d x=c, I_{\varepsilon}(u)<\infty\right\}$.

Here, $W^{1,1}(\Omega)$ denotes the Sobolev space of functions mapping $\Omega$ into $\mathbf{R}$ with integrable generalized derivative.

In this paper we shall study the following problem: If $u_{\varepsilon}$ is a solution of $\left(P_{\varepsilon}\right)$ and $u_{\varepsilon} \stackrel{L^{1}}{\rightarrow} u$ as $\varepsilon \rightarrow 0$, then what variational problem does $u$ satisfy? By our convexity (H4) and growth assumptions (H3) on $f, I_{\varepsilon}$ is weakly lower semicontinuous and

Received by the editors January 25, 1987 and, in revised form, September 20, 1987.

1980 Mathematics Subject Classification (1985 Revision). Primary 34D15; Secondary 82A25.

Research partially supported by National Science Foundation Grant DMS 8600710 under the Center for Applied Mathematics, Purdue University and National Science Foundation Grant DMS 8701448. 
minimizing sequences are compact, so $\left(P_{\varepsilon}\right)$ will have at least one solution. We shall show that $u$ satisfies the following classical minimal surface problem.

$$
\begin{array}{r}
\text { Minimize } \operatorname{Per}_{\Omega}\{u=a\} \text { over } u \in B V(\Omega) \text { with } \\
f(0, u(x))=0 \text { a.e. and } \int_{\Omega} u d x=c,
\end{array}
$$

(see $\S 1$ for the notation).

To see why we expect $u$ to satisfy $(M)$, we set $\varepsilon=0$ in the integrand of $I_{\varepsilon}$ and consider the variational problem:

$\left(\mathrm{P}_{0}\right)$ Minimize $I_{0}(u)=\int_{\Omega} f(0, u) d x$ on $\left\{u \in L^{1}(\Omega): \int_{\Omega} u d x=c, I_{0}(u)<\infty\right\}$.

Since $f(0, u)$ is nonconvex (H5) we expect solutions of $\left(\mathrm{P}_{0}\right)$ to be badly behaved. Let $S_{1}, S_{2}$ be any measurable sets such that $S_{1} \cup S_{2}=\Omega, S_{1} \cap S_{2}=\varnothing$ and $a\left|S_{1}\right|+b\left|S_{2}\right|=c|\Omega|$. Clearly, any function of the form

$$
y(x)= \begin{cases}a, & x \in S_{1}, \\ b, & x \in S_{2},\end{cases}
$$

is a solution of $\left(\mathrm{P}_{0}\right)$; that is, $\left(\mathrm{P}_{0}\right)$ has infinitely many piecewise constant solutions. We show now that $I_{\varepsilon}$ can be thought of as a singular perturbation of $I_{0}$. Expanding $f(\varepsilon|\nabla u|, u)$ in a Taylor series about $(0, u)$ and, noting that (H2) implies that the linear term vanishes, we have

$$
f(\varepsilon|\nabla u|, u)=f(0, u)+\frac{1}{2} f_{p p}(\varepsilon \theta|\nabla u|, u) \varepsilon^{2}|\nabla u|^{2}
$$

for some measurable function $\theta$ with $\theta(x) \in[0,1]$ a.e. By (H3), we see that $f(\varepsilon|\nabla u|, u)$ will be large for large values of $|\nabla u|$. Solutions of $\left(\mathrm{P}_{\varepsilon}\right)$ will be continuous and for $\varepsilon$ small we would expect these solutions to be close to a solution of $\left(\mathrm{P}_{0}\right)$; that is, $u_{\varepsilon}$ will take nearly the values $a$ and $b$ on the sets $S_{1}$ and $S_{2}$, respectively, except near the boundary where there will be an interface in which $\left|\nabla u_{\varepsilon}\right|$ takes large values. From above, the energy $I_{\varepsilon}$ will increase in these interfaces; the amount of increase being proportional to the area of the interface. Since we are assuming $u_{\varepsilon}$ is a minimizer of $I_{\varepsilon}, u_{\varepsilon}$ will be "close" to the solution of $\left(\mathrm{P}_{0}\right)$ which has the minimum interfacial area; this solution is given by $(\mathrm{M})$.

An alternative way of looking at this problem is in terms of the $\Gamma$-convergence of functionals (see the work of DeGiorgi [4], Modica and Mortola [10] and Attouch $[2])$.

The one-dimensional version of this problem is studied in Owen [11] (also see Carr, Gurtin and Slemrod [3]), in the context of a one-dimensional nonlinear elasticity model (Antman [1]) to predict nonhomogeneous behavior in elastic rods under tension (the small parameter, $\varepsilon$, is the undeformed radius). In this case, the EulerLagrange equation of the total energy are ordinary differential equations, which can be analyzed in detail and it is possible to find the pointwise limit of the minimizer as $\varepsilon \rightarrow 0$. The methods do not work for the two-dimensional case.

In Sternberg [12], the special case $f(p, u)=W(u)+p^{2}$ is studied, where $W(u)$ has the properties described in (H4) and (H5). This form of $f$ arises from the Van der Waals-Korteweg de Vries theory of phase transitions in a fluid: $u$ is the fluid density, $W(u)$ is the free-energy capable of supporting two phases and $\varepsilon^{2}|\nabla u|^{2}$ is 
a higher-order term representing lower order effects. Studying the behaviour of minimizers of the perturbed problem as $\varepsilon \rightarrow 0$ is a selection criterion (Gurtin [8]) to resolve nonuniqueness in the lower order problem (for an alternative selection criterion see Gurtin [7]).

Sternberg establishes the same result as ours, namely; the $L^{1}$ limit of minimizers satisfies (M). Our work, combined with that in Sternberg's [12], shows that there is no loss in generality in studying the behaviour of the simplest perturbations $\varepsilon^{2}|\nabla u|^{2}$ of $W(u)$ as a selection criterion as opposed to taking a more complicated perturbation.

The organization of this paper is as follows: In $\S 1$ we present some technical preliminaries concerning functions of bounded variation and sets of finite perimeter, and the statement of the main result (Theorem 1). $\S 2$ contains the proof the main lemma used in proving Theorem 2 . In $\S 3$, we discuss the compactness of the sequence of minimizers $u_{\varepsilon}$.

Many of the ideas used in this note are adapted from Sternberg [12] and Modica [9].

1. Technical preliminaries and statement of the result. In the first part of this section we state the results from the theory of functions of bounded variation which we shall need in $\S 2$. We shall also state an approximation theorem for sets of finite perimeter and a result for the signed distance function.

Let $\Delta$ be an open, bounded subset of $\mathbb{R}^{n}$ with Lipschitz continuous boundary. A function $u \in L^{1}(\Delta)$ belongs to the space of functions of bounded variation, $B V(\Delta)$, if

$$
\int_{\Delta}|\nabla u| \stackrel{\text { def }}{=} \sup _{\substack{g \in C_{0}^{1}\left(\Omega ; \mathbb{R}^{n}\right) \\|g| \leq 1}} \int u(x)(\nabla \cdot g(x)) d x<\infty .
$$

It follows that if $v_{\varepsilon} \rightarrow v$ in $L^{1}(\Delta)$, then

$$
\varliminf_{\varepsilon \rightarrow 0} \int_{\Delta}\left|\nabla u_{\varepsilon}\right| \geq \int_{\Delta}|\nabla u|
$$

$B V(\Delta)$ is a Banach space under the norm

$$
\|u\|_{B V(\Delta)}=\int_{\Delta}|u| d x+\int_{\Delta}|\nabla u| .
$$

For further details on $B V$ functions see Guisti [6].

If $A$ is a subset of $\mathbb{R}^{n}$ and $\chi_{A}$ is the characteristic function $A$, then $A$ is called a set of finite perimeter in $\Delta$ if

$$
\operatorname{Per}_{\Delta} A \stackrel{\text { def }}{=} \int_{\Delta}\left|\nabla \chi_{A}\right|=\sup _{\substack{g \in C_{0}^{1}\left(\Omega ; \mathbb{R}^{n}\right) \\|g| \leq 1}} \int_{A} \nabla \cdot g(x) d x<\infty .
$$

If $\partial A$ is smooth, the divergence theorem implies that

$$
\int_{\Delta}\left|\nabla \chi_{A}\right|=H^{n-1}(\partial A \cap \Delta)
$$

where $H^{n-1}$ is the $n-1$ dimensional Hausdorff measure. Thus, the perimeter of $A$ in $\Delta$ is a generalization of the surface area measure of the set $A$ lying in $\Delta$.

We shall need the following result from Sternberg [12]. It shows that any set of finite perimeter can be approximated by sets with smooth boundaries. 
LEMMA 1. Let $A \subset \Delta$ be a set of finite perimeter in $\Delta$ with $0<|A|<|\Delta|$. There exists a sequence of open sets $\left\{A_{k}\right\}$ satisfying the following:

(i) $\partial A_{k} \cap \Delta \in C^{2}$,

(ii) $\left|\left(\left(A_{k} \cap \Delta\right) \backslash A\right) \cup\left(A \backslash\left(A_{k} \cap \Delta\right)\right)\right| \rightarrow 0$ as $k \rightarrow \infty$,

(iii) $\operatorname{Per}_{\Delta} A_{k} \rightarrow \operatorname{Per}_{\Delta} A$ as $k \rightarrow \infty$,

(iv) $H^{n-1}\left(\partial A_{k} \cap \partial \Delta\right)=0$,

(v) $\left|A_{k} \cap \Delta\right|=|A|$ for $k$ sufficiently large.

For a set with a smooth boundary we can define a smooth signed distance function, measuring the distance from the boundary surface to a nearby point. The following result is again taken from Sternberg [12].

LEMMA 2. Let $B$ be a open subset of $\mathbb{R}^{n}$ with a $C^{2}$, compact, nonempty boundary such that $H^{n-1}(\partial B \cap \partial \Delta)=0$. Define the signed distance function to $\partial B$ by

$$
d(x)= \begin{cases}\operatorname{dist}(x, B), & x \in \Delta \backslash B, \\ -\operatorname{dist}(x, B), & x \in B \cap \Delta .\end{cases}
$$

Then, $d: \Delta \rightarrow \mathbb{R}$ and, for sufficiently small $s>0, d$ is a $C^{2}$ function with

$$
|\nabla d|=1 \quad \text { on }\{x:|d(x)|<s\} .
$$

Furthermore,

$$
\lim _{s \rightarrow 0} H^{n-1}(\{x: d(x)=s\})=H^{n-1}(\partial B) .
$$

Finally, we shall need the coarea formula (see Federer [5]):

$$
\int_{\Delta} k(h(x))|\nabla h| d x=\int_{-\infty}^{\infty} k(s) H^{n-1}\{x: h(x)=s\} d s
$$

for any measurable function $k$ and Lipschitz function $h$.

The main result of this paper is the following:

THEOREM 1. Let $u_{\varepsilon}$ be a solution of $\left(P_{\varepsilon}\right)$. If $u_{\varepsilon} \stackrel{L^{1}(\Omega)}{\longrightarrow} u_{0}$ as $\varepsilon \rightarrow 0$, then $u_{0}$ is a solution of

$$
\begin{gathered}
\text { Minimize } \operatorname{Per}_{\Omega}\{u=a\} \\
\text { on } \mathscr{C}=\left\{u \in B V(\Omega): f(0, u(x))=0 \text { a.e., } \int_{\Omega} u(x) d x=c\right\} .
\end{gathered}
$$

To prove this result we need first to construct a scalar function and then rescale the functional $I_{\varepsilon}$.

Consider the algebraic equation

$$
g(p, u) \stackrel{\text { def }}{=} f(p, u)-p f_{p}(p, u)=0 .
$$

From (H1), (H4), (H5) and (H6), we have $g(0, u) \geq 0, \lim _{p \rightarrow \infty} g(p, u) \leq 0$ and $g_{p}(p, u)<0$ for $p>0$. Thus, for each $u$ there is a unique $p=\hat{p}$ such that (1.4) is satisfied; that is, we have defined a scalar function $l: \mathbb{R} \rightarrow[0, \infty)$ by $l(u)=$ $\hat{p}$. It follows from (H5) that $l(u)=0$ if and only if $u=a$ or $b$. The implicit function theorem, $\mathrm{H} 4$ and a bootstrap argument imply then that $l(u)=C^{2}((0, l))$. Furthermore, $\left|l^{\prime}(u)\right|=\infty$ at $u=a$ or $b$. 
The function $l$ has a simple geometrical interpretation; if you fix $u$ and draw the "cross section" of the function $f(p, u)$ as a function of $p$, then $p=l(u)$ is the point where the tangent line which passes through the origin touches $f(p, u)$. The equation of this tangent line is

$$
y=f_{p}(\hat{p}, u) p=\frac{f(\hat{p}, u) p}{\hat{p}}
$$

We define a number $K$ to be

$$
K=\int_{a}^{b} \frac{f(l(\tau), \tau)}{l(\tau)} d \tau
$$

(since $\lim _{s \rightarrow 0} f(s, u) / s=0$ for all $u, K$ is finite).

In the case that $f(p, u)=W(u)+p^{2}$ (Sternberg [12]), we can compute $K$ explicitly. Substituting $W(u)+p^{2}$ into (1.4) gives

$$
l(u)=\sqrt{W(u)}
$$

so that

$$
K=\int_{a}^{b} \frac{W(\tau)+l(\tau)^{2}}{l(\tau)} d \tau=\int_{a}^{b} 2 \sqrt{W(\tau)} d \tau
$$

It is straightforward to construct piecewise linear functions $z_{\varepsilon}$ such that $I_{\varepsilon}\left(z_{\varepsilon}\right)=$ $O(\varepsilon)$ as $\varepsilon \rightarrow 0$. This suggests that to isolate the behaviour of $I_{\varepsilon}$ as $\varepsilon \rightarrow 0$ we scale $I_{\varepsilon}$ as follows. Let

$$
J_{\varepsilon}(u)=\left\{\begin{array}{l}
\int_{\Omega} \varepsilon^{-1} f(\varepsilon|\nabla u|, u) d x, \quad u \in W^{1,1}(\Omega), \int_{\Omega} u=c \\
+\infty \text { otherwise }
\end{array}\right.
$$

and

$$
J_{0}(u)=\left\{\begin{array}{l}
K \operatorname{Per}_{\Omega}\{x: u(x)=a\}, \quad u \in B V(\Omega), f(0, u(x))=0 \text { a.e., } \int_{\Omega} u=c, \\
+\infty \text { otherwise. }
\end{array}\right.
$$

Note, we have extended the definition of the scaled functionals so that $J_{\varepsilon}(u)$ and $J_{0}(u)$ are both defined on the same space $L^{1}(\Omega)$.

Theorem 2 will follow from the following lemma:

LEMMA 3. (i) For each $v \in L^{1}(\Omega)$ and for each sequence $\left\{v_{\varepsilon}\right\}$ in $L^{1}(\Omega)$ such that $v_{\varepsilon} \stackrel{L^{1}}{\rightarrow} v$ as $\varepsilon \rightarrow 0$,

$$
\varliminf_{\varepsilon \rightarrow 0} J_{\varepsilon}\left(v_{\varepsilon}\right) \geq J_{0}(v)
$$

(ii) For each $w \in L^{1}(\Omega)$, there exists a sequence $\left\{w_{\varepsilon}\right\}$ in $L^{1}(\Omega)$ such that $w_{\varepsilon} \stackrel{L^{1}}{\rightarrow} w$ as $\varepsilon \rightarrow 0$ and

$$
\lim _{\varepsilon \rightarrow 0} J_{\varepsilon}\left(w_{\varepsilon}\right)=J_{0}(w) .
$$

The proof of this lemma will be given in $\S 2$.

PROOF OF THEOREM 1. From (i) of Lemma 3 and our assumptions on $u_{\varepsilon}$ and $u_{0}$ we have

$$
\varliminf_{\varepsilon \rightarrow 0} J_{\varepsilon}\left(u_{\varepsilon}\right) \geq J_{0}\left(u_{0}\right) .
$$


Choose $w \in L^{1}(\Omega)$. Since $u_{\varepsilon}$ is a minimizer of $I_{\varepsilon}$ (and hence $J_{\varepsilon}$ ), there is, from (ii) of Lemma 3, a sequence of functions $\left\{w_{\varepsilon}\right\}$ in $L^{1}(\Omega)$ such that $w_{\varepsilon} \stackrel{L^{1}}{\rightarrow} w$ as $\varepsilon \rightarrow 0$ and

$$
J_{0}(w)=\varliminf_{\varepsilon \rightarrow 0} J_{\varepsilon}\left(w_{\varepsilon}\right) \geq \underline{\lim }_{\varepsilon \rightarrow 0} J_{\varepsilon}\left(u_{\varepsilon}\right) \geq J_{0}\left(u_{0}\right) .
$$

Since $w$ is arbitrary we conclude that $u_{0}$ is a minimizer of $J_{0}$. The result follows from the form of $J_{0}$.

The number $K$ is the energy left on the boundary as the boundary layer goes to zero.

Lemma 3 says that $J_{\varepsilon}$ converges to $J_{0}$ in the sense of $\Gamma$-convergence (see [4]). For more details on convergence of functionals see the book by Attouch [2], where $\Gamma$-convergence is referred to as epi-convergence.

2. Proof of Lemma 3. To prove (i) and (ii) we need only consider sequences $\left\{v_{\varepsilon}\right\}$ in $W^{1,1}(\Omega)$ satisfying $\int_{\Omega} v_{\varepsilon} d x=c$ and limit functions $v$ in $B V(\Omega)$ satisfying $\int_{\Omega} v d x=c$ and $f(0, v(x))=0$ a.e. For sequences and limits not satisfying these restrictions (i) and (ii) follow immediately from the definitions of $J_{\varepsilon}$ and $J_{0}$.

PROOF OF (i). Firstly, suppose $\left\{v_{\varepsilon}\right\}$ is a sequence in $L^{1}(\Omega)$ satisfying the uniform bound

$$
a \leq v_{\varepsilon} \leq b \quad \text { a.e. }
$$

Fix $u$ and let

$$
h(p)=f(p, u)-\frac{f(l(u), u)}{l(u)} p .
$$

It is simple to show that $h(0) \geq 0, h(l(u))=h^{\prime}(l(u))=0$ and $h^{\prime \prime}(p)>0$ for all $p$, which implies $h(p) \geq 0$ for all $p$.

Hence, we have

$$
J_{\varepsilon}\left(v_{\varepsilon}\right) \geq \int_{\Omega} \varepsilon^{-1} \frac{f\left(l\left(v_{\varepsilon}\right), v_{\varepsilon}\right)}{l\left(v_{\varepsilon}\right)} \cdot \varepsilon\left|\nabla v_{\varepsilon}\right| d x=\int_{\Omega}\left|\nabla \psi\left(v_{\varepsilon}\right)\right| d x
$$

where

$$
\psi(\tau)=\int_{a}^{\tau} \frac{f(l(s), s)}{l(s)} d s .
$$

For each $\varepsilon>0$, the function $\psi\left(v_{\varepsilon}(x)\right) \in B V(\Omega)$.

Suppose $v_{\varepsilon} \stackrel{L^{1}}{\rightarrow} v$. By the uniform bound (2.1), dominated convergence implies that $\psi\left(v_{\varepsilon}\right) \stackrel{L^{1}}{\rightarrow} \psi(v)$ as $\varepsilon \rightarrow 0$. We use then lower semicontinuity to deduce that

$$
\varliminf_{\varepsilon \rightarrow 0} J_{\varepsilon}\left(v_{\varepsilon}\right) \geq \varliminf_{\varepsilon \rightarrow 0} \int_{\Omega}\left|\nabla \psi\left(v_{\varepsilon}\right)\right| d x \geq \int_{\Omega}|\nabla \psi(v)| .
$$

Since $v$ only takes the values $a$ or $b$ (modulo a set of measure zero), it follows that

$$
\psi(v(x))= \begin{cases}0, & \{v=a\} \\ K, & \{v=b\} .\end{cases}
$$

Here, $\{v=a\}$ and $\{v=b\}$ denote the sets $\{x \in \Omega: v(x)=a\}$ and $\{x \in \Omega: v(x)=$ $b\}$, respectively. 
Therefore, $\psi(v)=K_{\chi\{v=b\}}$ and

$$
\int_{\Omega}|\nabla \psi|=K \operatorname{Per}_{\Omega}\{v=b\}=K \operatorname{Per}_{\Omega}\{v=a\}=J_{0}(v) .
$$

To remove the restriction $(2.1)$, set

$$
v_{\varepsilon}^{*}(x)= \begin{cases}a, & \left\{a>v_{\varepsilon}\right\}, \\ v_{\varepsilon}(x), & \left\{a \leq v_{\varepsilon} \leq b\right\}, \\ b, & \left\{v_{\varepsilon}>b\right\},\end{cases}
$$

so that $v_{\varepsilon}^{*}$ satisfies (2.1). If $v_{\varepsilon} \stackrel{L^{1}}{\rightarrow} v$ as $\varepsilon \rightarrow 0$, then $v_{\varepsilon}^{*} \stackrel{L^{1}}{\rightarrow} v$ (since $v$ takes only values $a$ or $b$ ). Furthermore, (H4), (H5) and (H6) imply $J_{\varepsilon}\left(v_{\varepsilon}\right) \geq J_{\varepsilon}\left(v_{\varepsilon}^{*}\right)$. From (2.3) and (2.4) we conclude that

$$
\varliminf_{\varepsilon \rightarrow 0} J_{\varepsilon}\left(v_{\varepsilon}\right) \geq \varliminf_{\varepsilon \rightarrow 0} J_{\varepsilon}\left(v_{\varepsilon}^{*}\right) \geq J_{0}(v) .
$$

Of course, $v_{\varepsilon}^{*}$ will not, in general, satisfy the integral constraint on $v$. However, this is unimportant since we have not used this constraint in deriving (2.3) and (2.4).

This completes the proof of (i).

Proof of (ii). Let $v \in L^{1}(\Omega)$ be a function such that $J_{0}(v)<\infty$ (so $v$ takes either of the values $a$ or $b$ ). Let $S_{1}$ denote the set where $v=a$ and $S_{2}$ the set where $v=b$. We shall construct a sequence of functions $v_{\varepsilon} \in W^{1,1}(\Omega)$ which converge to $v$ in $L^{1}$ and whose energies $J_{\varepsilon}\left(v_{\varepsilon}\right)$ converge to the energy $J_{0}(v)$. One can think of this sequence as a sequence of best approximations to the function $v$ and its energy $J_{0}(v)$.

To construct the sequence we first consider the ordinary differential equation

$$
q^{\prime} f_{p}\left(q^{\prime}, q\right)=f\left(q^{\prime}, q\right), \quad q(0)=(a+b) / 2 .
$$

By the definition of $l$ we can write (2.5) in the form

$$
q^{\prime}=l(q), \quad q(0)=(a+b) / 2 .
$$

Since $l$ is Lipschitz in a neighborhood of $(a+b) / 2$ we can apply standard results to solve (2.6) locally. Rewriting $(2.6)_{1}$ in the form

$$
\int_{(a+b) / 2}^{q(s)} \frac{d \tau}{l(\tau)}=s,
$$

and recalling that $l(\tau)>0$ for $a<\tau<b$, implies that the local solution of (2.6) can be extended to all $s \in(-\infty, \infty)$. Furthermore,

$$
\begin{array}{cc}
a<q(s)<b, & s \in(-\infty, \infty), \\
\lim _{s \rightarrow-\infty} q(s)=a, & \lim _{s \rightarrow+\infty} q(s)=b .
\end{array}
$$

By expanding $(l(\tau))^{2}$ in a Taylor series about $\tau=a$ we deduce from (H1), (H2), (H4), (H5) and the definition of $l$ that

$$
1 / l(\tau) \leq c_{1} /|\tau-a|,
$$

for $|\tau-a|$ sufficiently small, where $c_{1}>0$ is a constant. 
Likewise, for $|\tau-b|$ sufficiently small, there is a constant $c_{2}>0$ such that

$$
1 / l(\tau) \leq c_{2} /|\tau-b| .
$$

It follows then that the solution $q(s)$ of $(2.6)$ satisfies

$$
\begin{aligned}
& |q(s)-a| \leq \alpha e^{-\beta s} \quad \text { as } s \rightarrow-\infty, \\
& |q(s)-b| \leq \alpha e^{-\beta s} \quad \text { as } s \rightarrow+\infty,
\end{aligned}
$$

where $\alpha$ and $\beta$ are positive constants.

To construct the sequence of functions $v_{\varepsilon}$ we shall first construct a one-dimensional version of the sequence using $q(s)$.

Assume that the curve $\Gamma\left(=\partial S_{1} \cap \partial S_{2}\right)$ is $C^{2}$ (this assumption will be relaxed at the end of the proof).

Define a sequence of functions $g_{\varepsilon}: \mathbb{R} \rightarrow \mathbb{R}$ by

$$
g_{\varepsilon}(s)= \begin{cases}a, & s<-2 \varepsilon^{1 / 2}, \\ \left(\frac{q\left(-\varepsilon^{-1 / 2}\right)-a}{\varepsilon^{1 / 2}}\right)\left(s+2 \varepsilon^{1 / 2}\right)+a, & -2 \varepsilon^{1 / 2} \leq s<-\varepsilon^{1 / 2}, \\ q\left(\varepsilon^{-1} s\right), & -\varepsilon^{1 / 2} \leq s<\varepsilon^{1 / 2}, \\ \left(\frac{b-q\left(\varepsilon^{-1 / 2}\right)}{\varepsilon^{1 / 2}}\right)\left(s-2 \varepsilon^{1 / 2}\right)+b, & \varepsilon^{1 / 2} \leq s<2 \varepsilon^{1 / 2}, \\ b, & 2 \varepsilon^{1 / 2}<s,\end{cases}
$$

and a sequence of functions $\hat{v}_{\varepsilon}: \Omega \rightarrow \mathbb{R}$ by

$$
\hat{v}_{\varepsilon}(x)= \begin{cases}a, & \left\{x: d(x)<-2 \varepsilon^{1 / 2}\right\}, \\ g_{\varepsilon}(d(x)), & \left\{x:|d(x)|<2 \varepsilon^{1 / 2}\right\}, \\ b, & \left\{x: 2 \varepsilon^{1 / 2}<d(x)\right\},\end{cases}
$$

where $d(x)$ is the distance function defined in $\S 1$ with $\Delta=\Omega_{1}$ and $B=S_{1}$.

By Lemma 2, for $\varepsilon$ sufficiently small, $g_{\varepsilon}(d(x))$ will be absolutely continuous on $\left\{x:|d(x)|<2 \varepsilon^{1 / 2}\right\}$ and so $\hat{v}_{\varepsilon} \in W^{1,1}(\Omega)$. We show that $\hat{v}_{\varepsilon} \stackrel{L^{1}}{\rightarrow} v$. From the construction of $\hat{v}_{\varepsilon}$ we have (2.10)

$$
\begin{aligned}
\int_{\Omega}\left(\hat{v}_{\varepsilon}-v\right) d x= & \varepsilon^{-1 / 2} \int_{\left\{x:-\varepsilon^{1 / 2} \leq d<-\varepsilon^{1 / 2}\right\}}\left(q\left(-\varepsilon^{-1 / 2}\right)-a\right)\left(d(x)+2 \varepsilon^{1 / 2}\right) d x \\
& +\int_{\left\{x:-\varepsilon^{1 / 2} \leq d<0\right\}}\left(q\left(\varepsilon^{-1} d(x)\right)-a\right) d x \\
& +\int_{\left\{x: 0 \leq d<\varepsilon^{1 / 2}\right\}}\left(q\left(\varepsilon^{-1} d(x)\right)-b\right) d x \\
& +\int_{\left\{x: \varepsilon^{1 / 2} \leq d<2 \varepsilon^{1 / 2}\right\}}\left(b-q\left(\varepsilon^{-1 / 2}\right)\right)\left(d(x)-2 \varepsilon^{1 / 2}\right) d x .
\end{aligned}
$$

Consider the first integral in the right-hand side of (2.10); from the decay estimate (2.9) this term is $O\left(e^{\beta \varepsilon^{-1 / 2}}\right)$ as $\varepsilon \rightarrow 0$. 
Using the coarea formula (1.3) (with $n=2$ ) and (1.1) we can estimate the second integral on the right-hand side of $(2.10)$ by

$$
\begin{aligned}
\int_{\left\{x:-\varepsilon^{1 / 2} \leq d<0\right\}}\left(q\left(\varepsilon^{-1} d(x)\right)-a\right)|\nabla d| d x \\
\quad=\int_{-\varepsilon^{1 / 2}}^{0}\left(q\left(\varepsilon^{-1 / 2} s\right)-a\right) H^{1}\{d(x)=s\} d s \\
\quad \leq\left[\max _{-\varepsilon^{1 / 2} \leq s \leq 0} H^{1}\{d(x)=s\}\right] \int_{-\varepsilon^{1 / 2}}^{0}\left|q\left(\varepsilon^{-1 / 2} s\right)-a\right| d s \\
=\left[\max _{-\varepsilon^{1 / 2} \leq s \leq 0} H^{1}\{d(x)=s\}\right] \int_{-\varepsilon^{-1 / 2}}^{0}|q(s)-a| d s .
\end{aligned}
$$

From (1.2) and (2.9) we conclude that for $\varepsilon$ sufficiently small this term is less than

$$
C \varepsilon \int_{-\infty}^{0} e^{\beta \tau} d \tau, \quad C>0
$$

Similar estimates hold for the remaining terms in the right-hand side of $(2.10)$ so that

$$
\int_{\Omega}\left(\hat{v}_{\varepsilon}-v\right) d x=0(\varepsilon) \quad \text { as } \varepsilon \rightarrow 0
$$

that is $\hat{v}_{\varepsilon}$ converges to $v$ in $L^{1}$.

Next, we show that

$$
\lim _{\varepsilon \rightarrow 0} \int_{\Omega} \varepsilon^{-1} f\left(\varepsilon\left|\nabla v_{\varepsilon}\right|, v_{\varepsilon}\right) d x \leq J_{0}(v) .
$$

It is important to note that we have not written $\lim _{\varepsilon \rightarrow 0} J_{\varepsilon}\left(\hat{v}_{\varepsilon}\right)$ on the left-hand side of (2.13). This is because $\hat{v}_{\varepsilon}$ will, in general, not satisfy the constraint $\int_{\Omega} \hat{v}_{\varepsilon} d x=c$, so that $J_{\varepsilon}\left(\hat{v}_{\varepsilon}\right)=\infty$. It would then be impossible to establish an estimate of the type (2.13). We shall show later that it is possible to perturb $\hat{v}_{\varepsilon}$ slightly (without changing the convergence properties of the sequence) to satisfy the integral constraint.

By (H5), (1.1), (1.3) and the definition of $\hat{v}_{\varepsilon}$ we have, for $\varepsilon$ sufficiently small, (2.14)

$$
\begin{aligned}
\int_{\Omega} \varepsilon^{-1} f\left(\varepsilon\left|\nabla \hat{v}_{\varepsilon}\right|, \hat{v}_{\varepsilon}\right) d x= & \int_{\left\{x:|d|<2 \varepsilon^{1 / 2}\right\}} \varepsilon^{-1} f\left(\varepsilon \mid \nabla \hat{v}_{\varepsilon}, \hat{v}_{\varepsilon}\right)|\nabla d| d x \\
= & \int_{-2 \varepsilon^{1 / 2}}^{-\varepsilon^{1 / 2}} \varepsilon^{-2} f\left(\varepsilon\left|g_{\varepsilon}^{\prime}(s)\right|, g_{\varepsilon}(s)\right) H^{1}\{d(x)=s\} d s \\
& +\int_{-\varepsilon^{1 / 2}}^{\varepsilon^{1 / 2}} \varepsilon^{-1} f\left(\varepsilon\left|\frac{d}{d s} q\left(\varepsilon^{-1} s\right)\right|, q\left(\varepsilon^{-1}\right)\right) H^{1}\{d(x)=s\} d s \\
& +\int_{\varepsilon^{1 / 2}}^{2 \varepsilon^{1 / 2}} \varepsilon^{-1} f\left(\varepsilon\left|g_{\varepsilon}^{\prime}(s)\right|, g_{\varepsilon}(s)\right) H^{1}\{d(x)=s\} d s .
\end{aligned}
$$


The first integral on the right-hand side is bounded by (2.15)

$$
\begin{aligned}
& {\left[\max _{-2 \varepsilon^{-1 / 2} \leq s \leq \varepsilon^{-1 / 2}} H^{1}\{d(x)=s\}\right]} \\
& \int_{-2 \varepsilon^{1 / 2}}^{-\varepsilon^{1 / 2}} \varepsilon^{-1} f\left(\varepsilon^{1 / 2}\left|q\left(-\varepsilon^{-1 / 2}\right)-a\right|,\left(\frac{q\left(-\varepsilon^{-1 / 2}\right)-a}{\varepsilon^{1 / 2}}\right)\left(s+2 \varepsilon^{1 / 2}\right)+a\right) d s .
\end{aligned}
$$

Expanding $f(p, u)$ in a Taylor series about $(0, a)$ shows that $(2.15)$ equals

$$
\begin{gathered}
{\left[\max _{-2 \varepsilon^{-1 / 2} \leq s \leq \varepsilon^{-1 / 2}} H^{1}\{d(x)=s\}\right] \int_{-2 \varepsilon^{1 / 2}}^{-\varepsilon^{1 / 2}}\left(q\left(-\varepsilon^{-1 / 2}\right)-a\right)^{2}} \\
\cdot\left\{\frac{1}{2} f_{p p}(\theta(s), \psi(s))+\varepsilon^{-1} f_{p u}(\theta(s), \psi(s))\left(s+2 \varepsilon^{1 / 2}\right)\right. \\
\left.+\frac{1}{2} \varepsilon^{-2} f_{u u}(\theta(s), \psi(s))\left(s+2 \varepsilon^{1 / 2}\right)^{2}\right\} d s
\end{gathered}
$$

where $\theta$ and $\psi$ are measurable functions such that

$$
\begin{array}{r}
0 \leq \theta(s) \leq \varepsilon^{1 / 2}\left|q\left(-\varepsilon^{-1 / 2}\right)-a\right|, \quad a \leq \psi(s) \leq \varepsilon^{-1 / 2}\left(q\left(-\varepsilon^{-1 / 2}\right)-a\right)\left(s+2 \varepsilon^{1 / 2}\right) \\
\forall s \in\left(-2 \varepsilon^{1 / 2},-\varepsilon^{1 / 2}\right) .
\end{array}
$$

The estimates (2.9) and the smoothness of $f$ then imply that this term goes to zero exponentially fast as $\varepsilon \rightarrow 0$.

Likewise, the last integral in (2.14) goes to zero as $\varepsilon \rightarrow 0$.

We consider now the remaining integral on the right-hand side of (2.14). This is less than or equal to

$$
\left[\max _{-\varepsilon^{1 / 2} \leq s \leq \varepsilon^{1 / 2}} H^{1}\{d(x)=s\}\right] \int_{-\varepsilon^{1 / 2}}^{\varepsilon^{1 / 2}} \varepsilon^{-1} f\left(q^{\prime}\left(\varepsilon^{-1} s\right), q\left(\varepsilon^{-1} s\right)\right) d s .
$$

Changing variables: $t=q\left(\varepsilon^{-1} s\right), d t=\varepsilon^{-1} q^{\prime}\left(\varepsilon^{-1} s\right) d s=\varepsilon^{-1} l\left(q\left(\varepsilon^{-1} s\right)\right)^{1 / 2} d s=$ $\varepsilon^{-1} l(t)^{1 / 2} d s$, and recalling that $q$ satisfies (2.5), shows that (2.16) equals

$$
\left[\max _{-\varepsilon^{1 / 2} \leq s \leq \varepsilon^{1 / 2}} H^{1}\{d(x)=s\}\right] \int_{q\left(-\varepsilon^{-1 / 2}\right)}^{q\left(\varepsilon^{-1 / 2}\right)} f\left(l(t)^{1 / 2}, t\right) l(t)^{-1 / 2} d t,
$$

and this is less than

$$
\begin{gathered}
{\left[\max _{-\varepsilon^{1 / 2} \leq s \leq \varepsilon^{1 / 2}} H^{1}\{d(x)=s\}\right] \int_{a}^{b} f\left(l(t)^{1 / 2}, t\right) l(t)^{-1 / 2} d t} \\
=\left[\max _{-\varepsilon^{1 / 2} \leq s \leq \varepsilon^{1 / 2}} H^{1}\{d(x)=s\}\right] K .
\end{gathered}
$$

Letting $\varepsilon \rightarrow 0$, it follows from (1.2), (2.14) and our assumption that $\Gamma$ is in $C^{2}$, that

$$
\lim _{\varepsilon \rightarrow 0} \int_{\Omega} \varepsilon^{-1} f\left(\varepsilon\left|\nabla \hat{v}_{\varepsilon}\right|, \hat{v}_{\varepsilon}\right) \leq K \operatorname{Per}_{\Omega}\{v=a\}=J_{0}(v) .
$$

As mentioned above, $\hat{v}_{\varepsilon}$ will not satisfy $\int_{\Omega} \hat{v}_{\varepsilon} d x=c$. Define a sequence of numbers $\eta_{\varepsilon}=|\Omega|^{-1}\left\{c-\int_{\Omega} \hat{v}_{\varepsilon} d x\right\}$. From (2.11),

$$
\eta_{\varepsilon}=O(\varepsilon) \quad \text { as } \varepsilon \rightarrow 0
$$


For $\varepsilon>0$, let

$$
v_{\varepsilon}(x)=\hat{v}_{\varepsilon}(x)+\eta_{\varepsilon}
$$

Clearly,

$$
v_{\varepsilon} \in W^{1,1}(\Omega), \quad v_{\varepsilon} \stackrel{L^{1}}{\rightarrow} v \quad \text { and } \quad \int_{\Omega} v_{\varepsilon}(x) d x=c
$$

We claim that

$$
\lim _{\varepsilon \rightarrow 0} J_{\varepsilon}\left(v_{\varepsilon}\right) \leq \lim _{\varepsilon \rightarrow 0} \int_{\Omega} \varepsilon^{-1} f\left(\varepsilon\left|\nabla \hat{v}_{\varepsilon}\right|, \hat{v}_{\varepsilon}\right) d x .
$$

It follows then from (2.13) and part (i) that

$$
\lim _{\varepsilon \rightarrow 0} J_{\varepsilon}\left(v_{\varepsilon}\right)=J_{0}(v)
$$

By the construction of $v_{\varepsilon}$, we see that

$$
\begin{aligned}
J_{\varepsilon}\left(v_{\varepsilon}\right)= & \varepsilon^{-1} f\left(0, a+\eta_{\varepsilon}\right)\left|\left\{\alpha: d(x) \leq-2 \varepsilon^{1 / 2}\right\}\right| \\
& +\int_{\left\{x:|d|<2 \varepsilon^{1 / 2}\right\}} \varepsilon^{-1} f\left(\varepsilon\left|\nabla \hat{v}_{\varepsilon}\right|, \hat{v}_{\varepsilon}+\eta_{\varepsilon}\right) d x \\
& +\varepsilon^{-1} f\left(0, b+\eta_{\varepsilon}\right)\left|\left\{x: d(x) \geq 2 \varepsilon^{1 / 2}\right\}\right| .
\end{aligned}
$$

By expanding $f(0, u)$ in a Taylor series about $(0, a)$ we obtain

$$
\varepsilon^{-1} f\left(0, a+\eta_{\varepsilon}\right)\left|\left\{x: d(x) \leq-2 \varepsilon^{1 / 2}\right\}\right| \leq \frac{1}{2} \varepsilon^{-1}|\Omega| f_{u u}\left(0, a+\gamma_{\varepsilon} \eta_{\varepsilon}\right) \eta_{\varepsilon}^{2}
$$

for some $0 \leq \gamma_{\varepsilon} \leq 1$. Since $\eta_{\varepsilon}$ behaves like $\varepsilon$ as $\varepsilon \rightarrow 0$ we deduce that the first term on the right-hand side of $(2.20)$ goes to zero as $\varepsilon \rightarrow 0$. Likewise, the third term in (2.20) also goes to zero with $\varepsilon$.

Hence, to establish (2.18) we must show

$$
\lim _{\varepsilon \rightarrow 0} \int_{\left\{x:|d|<2 \varepsilon^{1 / 2}\right\}} \varepsilon^{-1}\left\{f\left(\varepsilon\left|\nabla \hat{v}_{\varepsilon}\right|, \hat{v}_{\varepsilon}+\eta_{\varepsilon}\right)-f\left(\varepsilon\left|\nabla \hat{v}_{\varepsilon}\right|, \hat{v}_{\varepsilon}\right)\right\} d x \leq 0
$$

(we have used the fact that $f\left(\varepsilon\left|\nabla \hat{v}_{\varepsilon}(x)\right|, \hat{v}_{\varepsilon}(x)\right)=0$ for $|d(x)| \geq 2 \varepsilon^{1 / 2}$ ). By the mean value theorem, the integrand in $(2.21)$ equals $f_{u}\left(\varepsilon\left|\nabla \hat{v}_{\varepsilon}\right|, v_{\varepsilon}+\psi \eta_{\varepsilon}\right) \varepsilon^{-1} \eta_{\varepsilon}$ where $\psi$ is a measurable function with $\psi(x) \in[0,1]$ a.e. As $\varepsilon \rightarrow 0,\left|\nabla \hat{v}_{\varepsilon}\right|$ is $O\left(\varepsilon^{-1}\right)$ so the integrand is $O(1)$. Since $\left|\left\{x:|d|<2 \varepsilon^{1 / 2}\right\}\right| \rightarrow 0$ as $\varepsilon \rightarrow 0$ this proves (2.21) (with equality).

Thus, (ii) follows from (2.17) and (2.19) provided $\Gamma$ is in $C^{2}$. To remove this restriction we can use exactly the argument given in Sternberg [12]. Let $v \in B V(\Omega)$ satisfy $\int_{\Omega} v d x=c$ and

$$
v(x)= \begin{cases}a, & x \in S_{1} \\ b, & x \in \Omega \backslash S_{1}\end{cases}
$$

where $S_{1}$ is a set of finite perimeter. From Lemma 1 we can find a sequence $\left\{A_{k}\right\}$ of approximating sets. Let

$$
v_{k}(x)= \begin{cases}a, & x \in A_{k} \cap \Omega, \\ b, & x \in \Omega \backslash A_{k} .\end{cases}
$$

From property (iii) of Lemma 1 and the definition of $J_{0}$

$$
J_{0}\left(v_{k}\right) \rightarrow J_{0}(v) \quad \text { as } k \rightarrow \infty
$$


while property (ii) implies $v_{k} \stackrel{L^{1}}{\rightarrow} v$ as $k \rightarrow \infty$. Since $\partial A_{k}$ is $C^{2}$ we can find a sequence $\left\{v_{k, \varepsilon}\right\}$ in $W^{1,1}(\Omega)$ such that $v_{k, \varepsilon} \stackrel{L^{1}}{\rightarrow} v_{k}$ as $\varepsilon \rightarrow 0$ and

$$
\lim _{\varepsilon \rightarrow 0} J_{\varepsilon}\left(v_{k, \varepsilon}\right)=J_{0}\left(v_{k}\right) \text {. }
$$

We can now use a diagonalization argument to give a sequence $\left\{v_{k_{\varepsilon_{j}}, \varepsilon_{j}}\right\}$ in $W^{1,1}(\Omega)$ converging to $v$ in $L^{1}$ and with the energies converging. This completes the proof of (ii).

3. Compactness of $\left\{u_{\varepsilon}\right\}$. In [9 and 12], sufficient conditions have been given for the case $f(p, u)=W(u)+p^{2}$ to ensure that the sequence of minimizers, $\left\{u_{\varepsilon}\right\}$, of $I_{\varepsilon}$ contains an $L^{1}$-convergent subsequence.

For the general case considered in this paper, if $f(p, u)$ satisfies the reasonable growth condition (cf. $[\mathbf{9}, \mathbf{1 2}])$ :

There exist constants $e_{2} \geq e_{1}>0, \alpha \geq \beta \geq 2$ and $R>0$ such that

$$
e_{1}\left\{|s|^{\alpha}+p^{\beta}\right\} \leq f(p, u) \leq e_{2}\left\{|s|^{\alpha}+p^{\beta}\right\} \text { for }|u|+p>R,
$$

then it can easily be shown that there exist positive constants $C_{1} \leq C_{2}$ such that

$$
C_{1}|\tau|^{\alpha\left(1-\beta^{-1}\right)} \leq \frac{f(l(\tau), \tau)}{l(\tau)} \leq C_{2}|\tau|^{\alpha\left(1-\beta^{-1}\right)} \text { for }|\tau|>R .
$$

It follows by a simple adaption of the proof of Proposition 3 of Modica's paper [9] that the sequence $\left\{u_{\varepsilon}\right\}$ contains an $L^{1}$-convergent subsequence. We refer the reader to [9] for further details.

ACKNOWLedgment. The author would like to thank Robert Kohn and Peter Sternberg for helpful discussions.

\section{REFERENCES}

1. S. S. Antman, Nonuniqueness of equilibrium states for bars in tension, J. Math. Anal. Appl. 44 (1973), 333-349.

2. H. Attouch, Variational convergence for functions and operators, Pitman, 1984.

3. J. Carr, M. E. Gurtin and M. Slemrod, Structured phase transitions on a finite interval, Arch. Rational Mech. Anal. 86 (1984), 317-351.

4. E. DeGiorgi, Convergence problems for functionals and operators, Proc. Internat. Meeting on Recent Methods in Nonlinear Anal. (Rome, 1978), Pitagora, Bologna, 1979, pp. 131-188.

5. H. Federer, Gesmetric measure theory, Springer-Verlag, Berlin and New York, 1969.

6. E. Guisti, Minimal surfaces and functions of bounded variation, Birkhäuser, 1984.

7. M. E. Gurtin, On a theory of phase transitions with interfacial energy, Arch. Rational Mech. Anal. 87 (1984), 187-212.

8.

9. L. Modica, Gradient theory of phase transitions and minimal interface criteria, Arch. Rational Mech. Anal. 98 (1987), 123-142.

10. L. Modica and S. Mortola, Un esempio de $\Gamma$-convergenza de una famiglia de funzionali ellittici, Boll. Un. Mat. Ital. A14 (1977).

11. N. C. Owen, Existence and stability of necking deformations for nonlinearly elastic rods, Arch. Rational Mech. Anal. 98 (1987), 357-383.

12. P. Sternberg, The effect of a singular perturbation on nonconvex variational problems, Arch. Rational Mech. Anal. (to appear).

Department of Mathematics, Purdue University, West lafayette, Indiana 47907

Current address: School of Mathematical Sciences, University of Bath, Claverton Down, Bath, BAZ 7AY, U. K. 